\title{
Physician Stress Driven by Complex Patients
}

providers, and patients. Given the profound health consequences of social determinants, providing high-quality care to patients must also include meeting their social needs.

W e appreciate Dr. Kirschner's insightful comments and observations, and we agree with her recommendation that structural and systems-level interventions are necessary to meet the medical, behavioral health and social needs of complex patients. Our study highlights that without these resources the unmet needs of complex patients-not the patients themselves - can drive primary care physician (PCP) stress.

Dr. Kirschner also suggests that "moral injury" may be an important factor affecting PCPs caring for complex patients. Moral injury, or more broadly moral distress, is a phenomenon where healthcare providers feel a sense of responsibility and yet a sense of powerlessness caring for patients. ${ }^{1}$ While previous research suggests that the stress from a high volume of clerical tasks, excessive workloads, and corrosive organizational culture drives physician burnout, ${ }^{2,}{ }^{3}$ thought-leaders now propose that factors related to patient care are also relevant. In a recent editorial, Lee et al. describe a pathway to reduce burnout where clinicians maximize the inherent rewards of the job like meaningful conversations with patients or delivering high-quality care. ${ }^{4}$ The authors also differentiate between the inherent stress of working as a physician and the added stresses imposed by existing systems of care that can lead to moral distress.

Applying our study findings to this framework suggests that better meeting complex patients' social needs through interventions like care coordination or team-based care may lessen moral distress and reduce burnout. Physicians, however, cannot do this work alone. Successful systems-level efforts require engaging with healthcare system leaders, payers,

\section{REFERENCES}

1. Landry C. Not knowing the "right thing to do:" Moral distress and tolerating uncertainty in medicine. Clin Ethics. 2017;12(1):37-44. https://doi.org/10.1177/1477750916661978

2. West CP, Dyrbye LN, Shanafelt TD. (Mayo Clinic, Rochester, MN; and Stanford University Medical Center, Stanford, CA, USA). Physician burnout: contributors, consequences and solutions (Review). J Intern Med. 2018;283: 516- 529.

3. Rabatin J, et al. Predictors and Outcomes of Burnout in Primary Care Physicians. J Prim Care Community Health. 2016;7(1):41-43.

4. Lee TH, Mylod DE. Deconstructing Burnout to Define a Positive Path Forward. JAMA Intern Med. Published online February 04, 2019;179(3):429-430 